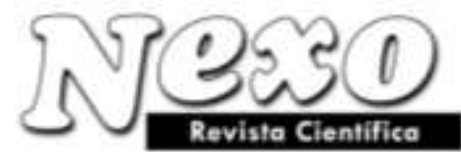

Vol. 30, No. 01, pp. 48-59/Junio 2017

\title{
FAMILIA DE SOLUCIONES ELEMENTALES DEL OPERADOR DE LA ONDA ITERADO K-VECES
}

\section{ELEMENTARY SOLUTION FAMILY OF THE WAVE OPERATION ITERAYED K-TIMES}

\author{
M. A. Aguirre* y E. A. Aguirre Rébora \\ Universidad Nacional del Centro, Facultad de Ciencias Exactas, Núcleo Consolidado Matemática Pura y \\ Aplicada (NuCOMPA), Tandil, Provincia de Buenos Aires, Argentina. \\ * manuel.aguirre48@gmail.com
}

(recibido/received: 09-Febrero-2017; aceptado/accepted: 10-Abril-2017)

\section{RESUMEN}

Consideremos el operdor $L_{t}=\frac{\partial^{2}}{\partial t^{2}}-c^{2} \Delta$, donde $\Delta$ es el operador Laplaciano definido por (22) y sea $L_{t}^{k}$ el operador de onda iterado $k$-veces definido por (21).En este artículo se obtiene familias de soluciones elementales $E_{k}(x, t)$ (ver fórmula (29) y $\overline{E_{k}(x, t)}$ (ver fórmula (54) del operador $L_{t}$ iterado $k$-veces, para los casos $n$ par y $n$ impar respectivamente. Como consecuencia se obtiene que $u_{k}(x, t)$ definida por (60) y $\left.V_{k}(x, t)\right)$ definida por (61) son soluciones de la ecuación diferencial no lineal $L_{t}^{k} u_{k}(x, t)=f\left(x, t, u_{k}(x, t)\right)$ para $k=1,2,3, \ldots$

Palabras Claves: Solución elemental; Operador de onda; Teoría de distribuciones.

\section{ABSTRACT}

Let $L_{t}=\frac{\partial^{2}}{\partial t^{2}}-c^{2} \Delta$ be wave operator, where $\Delta$ is the laplacian operator defined by(22) and let $L_{t}^{k}$ be the wave iterated $k$-times operator defined by(21). In this article we obtain the elementary solutions $E_{k}(x, t)$ (see formula (29) and $\overline{E_{k}(x, t)}$ (see formula (54) of the operator $L_{t}$ iterated $k$ times, for the cases $n$ even and $n$ odd respectively. As consequence we obtain that $u_{k}(x, t)$ defined by (60) and $\left.V_{k}(x, t)\right)$ defined by (61) are solutions of nolineardiferential equation $L_{t}^{k} u_{k}(x, t)=f\left(x, t, u_{k}(x, t)\right)$ for $\mathrm{k}=1,2,3 \ldots$

Keywords: Elementary solutions; Wave operation; Distributions Theory. 


\section{INTRODUCCIÓN}

Sea $(x, t)=\left(x_{1} x_{2}, \ldots x_{n}, t\right)$ un punto de $R^{n+1}$, donde $t>0$, y sea $P_{c}(x, t)$ definida por

$P_{c}(x, t)=c^{2} t^{2}-|x|^{2}$

donde:

$|x|^{2}=x_{1}^{2}+x_{2}^{2}+\cdots+x_{n}^{2}$

Por $\Gamma_{+}$designamos el interior del cono:

$\Gamma_{+}=\left\{(x, t) \in R^{n+1}, t>0, P_{c}(x, t)>0\right\}$

y con $\overline{\Gamma_{+}}$su clausura.

Se define la función generalizada $\left(P_{c}(x, t)\right)_{+}^{\lambda}$, usando el mismo método dado en (Gelfand y Shilov 1964), donde $\lambda$ es un número complejo, de la siguiente forma:

$<\left(P_{c}(x, t)\right)_{+,}^{\lambda}, \varphi(x, t)>=\int_{\Gamma_{+}} P_{c}(x, t) \varphi(x, t) d x d t=\int_{t>0} \int_{R}{ }_{n} P_{c}(x, t) \varphi(x, t) d x d t$

donde $\varphi(x, t)$ es una función de prueba en las variables $(x, t)$.

Por cálculos directos de (4) se tiene,

$<\left(P_{c}(x, t)\right)_{+,}^{\lambda}, \varphi(x, t)>=\int_{0}^{\infty} \xi^{\lambda+\frac{n}{2}-\frac{1}{2}} G(\lambda, \xi) d \xi$

donde

$G(\lambda, \xi)=\frac{1}{4 c} \int_{0}^{1}(1-h)^{\lambda} h^{\frac{n-2}{2}} \phi_{1}(\xi, \xi h) d h$,

$\phi_{1}(\xi, \xi h)=\phi(\xi, h)=\phi(t, \varrho)=\int_{\Omega_{n-1}} \varphi(\varrho \omega, t) d \Omega_{n-1}$

y $\Omega_{n-1}$ es el área de la hipersuperficie de la esfera unitaria dentro del espacio Euclideano (n-1) dimensional.

La fórmula (5) es similar a la fórmula (10) dada en (Gelfand y Shilov, 1964), para el caso $p=$ 1 у $q=n-1$.

Por tanto de Gelfand y Shilov página 255, el funcional $<\left(P_{c}(x, t)\right)_{+,}^{\lambda}, \varphi(x, t)>$ tiene dos grupos de singularidades, a saber $\lambda=-1,-2, \ldots y \lambda=-\frac{n}{2},-\frac{n}{2}-1, \ldots$

Por otra parte, tomando en cuenta la definición $<\delta^{(k)}\left(P_{c}(x, t)\right)_{+}, \varphi(x, t)>$

Nexo Revista Científica Vol. 30, No. 01, pp. 48-59/Junio 2017 
dada en (Gelfand y Shilov, 1964) y usando el mismo método dado en (Gelfand y Shilov, 1964), después de cálculos elementales se obtiene la siguiente fórmula:

$\operatorname{Res}_{\lambda=-k}\left(P_{c}(x, t)\right)_{+}^{\lambda}=\frac{(-1)^{k-1}}{(k-1) !} \delta^{(k)}\left(P_{c}(x, t)\right)_{+}$

si $n$ es par, la fórmula también es válida si $n$ es impar pero bajo la condición $k<\frac{n-1}{2}$.

De (Gelfand y Shilov, 1964), el funcional $\left\langle x_{+}^{\lambda}, \boldsymbol{\varphi}\right\rangle$ es regular para todo $\lambda$ excepto para $\lambda=-k$, $k=1,2,3, \ldots$ donde tiene polos simples y vale que

$\operatorname{Res}_{\lambda=-k}<x_{+}^{\lambda}, \boldsymbol{\varphi}>=\frac{1}{(k-1) !} \varphi^{(k-1)}(0)$.

Ahora de (5) y usando (10) se tiene,

$\operatorname{Res}_{\lambda=-\left(\frac{n+1}{2}\right)-k}<\left(P_{c}(x, t)\right)_{+,}^{\lambda}, \varphi(x, t)>=\frac{1}{k !}\left[\frac{\partial^{k}}{\partial \xi^{k}} G_{-\left(\frac{n+1}{2}\right)-k, \xi}\right]_{\xi=0}$

Si $n$ es par y $k=0,1,2, \ldots$

Haciendo $k=0$ en (11) se tiene,

$\operatorname{Res}_{\lambda=-\left(\frac{n+1}{2}\right)}<\left(P_{c}(x, t)\right)_{+,}^{\lambda}, \varphi(x, t)>=\left[G_{-\left(\frac{n+1}{2}\right), \xi}\right]_{\xi=0}=G_{-\left(\frac{n+1}{2}\right)}(0)$.

De (6) y usando la fórmula

$\int_{0}^{1}(1-t)^{\lambda} t^{\mu-1} d t=B(\lambda, \mu)=\frac{\Gamma(\lambda) \Gamma(\mu)}{\Gamma(\lambda+\mu)}$

Para $\operatorname{Re}(\boldsymbol{\lambda})>0$ y $\operatorname{Re}(\mu)>0$, donde $\boldsymbol{\Gamma}(\boldsymbol{\alpha})$ es la función gama. De (12) usando (6) y (13) se tiene

$G_{-\left(\frac{n+1}{2}\right)}(0)=\frac{\phi_{1}(0,0)}{4 c} \cdot \frac{\Gamma\left(-\frac{n}{2}+\frac{1}{2}\right) \Gamma\left(\frac{n}{2}\right)}{\Gamma\left(\frac{1}{2}\right)}$.

Ahora considerando que $\Omega_{d}$ es el área de la esfera unitaria en $R^{d}$ y está dada por la fórmula

$$
\Omega_{d}=\frac{2 \pi^{\frac{d}{2}}}{\Gamma\left(\frac{d}{2}\right)}
$$

donde $d$ es la dimensión del espacio y usando la fórmula

$\boldsymbol{\Gamma}(\mathbf{z}) \boldsymbol{\Gamma}(\mathbf{1}-\mathbf{z})=\frac{\pi}{\operatorname{sen}(\mathbf{z} \pi)}($ Erdelyi, 1953) 
se tiene

$$
G_{-\left(\frac{n+1}{2}\right)}(0)=\frac{\varphi(0,0)}{4 c} \cdot \frac{2 \pi^{\frac{n-1}{2}} \pi \Gamma\left(\frac{n}{2}\right)}{(-1)^{\frac{n}{2}} \Gamma\left(\frac{n-1}{2}\right) \Gamma\left(\frac{1}{2}\right) \Gamma\left(\frac{n+1}{2}\right)}
$$

si $n$ es par.

De (12), considerando (17) y usando que $\Gamma\left(\frac{1}{2}\right)=\sqrt[2]{\pi}$ y

$\varphi(0,0)=<\delta(\mathrm{x}, \mathrm{t}), \varphi(\mathrm{x}, \mathrm{t})>(18)$

se llega a la siguiente expresión

$\operatorname{Res}_{\lambda=-\left(\frac{n+1}{2}\right)}<\left(P_{c}(x, t)\right)_{+,}^{\lambda}, \varphi(x, t)>=<\frac{1}{2 c} \frac{\pi^{\frac{n}{2}} \pi \Gamma\left(\frac{n}{2}\right)}{\left.(-1)^{\frac{n}{2}} \Gamma\left(\frac{n-1}{2}\right)\right) \Gamma\left(\frac{n+1}{2}\right)} \delta(\mathrm{x}, \mathrm{t}), \varphi(\mathrm{x}, \mathrm{t})>$

De (19) se obtiene la siguiente fórmula:

$\operatorname{Res}_{\lambda=-\left(\frac{n+1}{2}\right)}\left(P_{c}(x, t)\right)_{+,}^{\lambda}=\frac{1}{2 c} \frac{\pi^{\frac{n}{2}} \pi \Gamma\left(\frac{n}{2}\right)}{\left.(-1)^{\frac{n}{2}} \Gamma\left(\frac{n-1}{2}\right)\right) \Gamma\left(\frac{n+1}{2}\right)} \delta(\mathrm{x}, \mathrm{t})$.

\section{FAMILIA DE SOLUCIONES ELEMENTALES DEL OPERADO DE LA ONDA ITERADO K-VECES}

Caso 1: $n$ par. Sea $L_{t}^{k}$ el operador de la onda iterado k veces, definido por

$L_{t}^{k}=\left\{\frac{\partial^{2}}{\partial t^{2}}-c^{2} \Delta\right\}^{k}$

donde $k=0,1,2, \ldots$ y $\Delta$ es el operador Laplaciano definido por,

$\Delta=\sum_{j=1}^{n} \frac{\partial^{2}}{\partial x_{j}^{2}}$

Vamos a encontrar una familia de funciones distribucionales $E_{k}(x, t) d e L_{t}^{k}$ para este caso.

Se sabe que una solución elemental $E_{k}(x, t) d e L_{t}^{k}$, es una solución de la ecuación

$L_{t}^{k} E_{k}(x, t)=\delta(x, t)$

Por otra parte, por cálculos elementales se obtiene la siguiente propiedad

$L_{t}^{k}\left(P_{c}(x, t)\right)_{+,}^{\lambda+1}=2 c^{2}(\boldsymbol{\lambda}+\mathbf{1})(\mathbf{2} \boldsymbol{\lambda}+\boldsymbol{n}+\mathbf{1})\left(P_{c}(x, t)\right)_{+,}^{\lambda}$ 
Haciendo $k$ iteraciones se obtiene

$L_{t}^{k}\left(P_{c}(x, t)\right)_{+,}^{\lambda+k}=\left(2 c^{2}\right)^{k}(\boldsymbol{\lambda}+\mathbf{1}) . .(\boldsymbol{\lambda}+\boldsymbol{k})(\mathbf{2} \boldsymbol{\lambda}+\boldsymbol{n}+\mathbf{1}) \ldots(\mathbf{2} \boldsymbol{\lambda}+\boldsymbol{n}+\mathbf{2} \boldsymbol{k}+\mathbf{1})\left(P_{c}(x, t)\right)_{+,}^{\lambda}$,

Usando la fórmula

$\frac{\Gamma(z)}{\Gamma(z-m)}=(\mathrm{z}-1) \ldots(\mathrm{z}-\mathrm{m}), \mathrm{m}=1,2, \ldots$

De (Erdelyi, 1953), la fórmula (25) puede ser escrita de la siguiente forma:

$L_{t}^{k}\left(P_{c}(x, t)\right)_{+,}^{\lambda+k}=\frac{2^{k}\left(c^{2}\right)^{k}(-\mathbf{1})^{k} \Gamma(-\lambda)}{\Gamma(\lambda-k)}[(2 \lambda+\boldsymbol{n}+\mathbf{1}) \ldots(2 \lambda+\boldsymbol{n}+\mathbf{2} \boldsymbol{k}+\mathbf{1})]\left(P_{c}(x, t)\right)_{+}^{\lambda}$

De (27), usando la fórmula (20) y tomando en cuenta que $\left(P_{c}(x, t)\right)_{+}^{\lambda}$, en $\lambda=\mathrm{k}-\left(\frac{n+1}{2}\right)$ es una función regular si $n$ es par, se obtiene

$$
\begin{aligned}
& L_{t}^{k}\left(P_{c}(x, t)\right)_{+,}^{\mathrm{k}-\left(\frac{n+1}{2}\right)}=\lim _{\lambda \mapsto-\left(\frac{n+1}{2}\right)} L_{t}^{k}\left(P_{c}(x, t)\right)_{+,}^{\lambda+k}=\lim _{\lambda \mapsto-\left(\frac{n+1}{2}\right)} \frac{2^{k}\left(c^{2}\right)^{k}(-1)^{k} \Gamma(-\lambda)}{\Gamma(\lambda-k)} . \\
& \lim _{\lambda \mapsto-\left(\frac{n+1}{2}\right)}[(2 \lambda+n+1) \ldots(2 \lambda+n+2 k-1)] . \\
& \lim _{\lambda \mapsto-\left(\frac{n+1}{2}\right)}(2 \boldsymbol{\lambda}+\boldsymbol{n}+\mathbf{1})\left(P_{c}(x, t)\right)_{+}^{\lambda}= \\
& =\frac{\mathbf{2}^{k}\left(c^{2}\right)^{k}(-1)^{k} \Gamma\left(\left(\frac{n+1}{2}\right)\right)}{\Gamma\left(\left(\frac{n+1}{2}\right)-k\right)}[2.4 .6 .8 \ldots 2 k] .2 \operatorname{Res}_{\lambda=-\left(\frac{n+1}{2}\right)}\left(P_{c}(x, t)\right)_{+,}^{\lambda}= \\
& =\frac{2^{k}\left(c^{2}\right)^{k}(-1)^{k} \Gamma\left(\left(\frac{n+1}{2}\right)\right)}{\Gamma\left(\left(\frac{n+1}{2}\right)-k\right)}[2.4 .6 .8 \ldots 2 k] .2 \frac{1}{2 c} \frac{\pi^{\frac{n}{2}} \pi \Gamma\left(\frac{n}{2}\right)}{\left.(-1)^{\frac{n}{2}} \Gamma\left(\frac{n-1}{2}\right)\right) \Gamma\left(\frac{n+1}{2}\right)} \delta(\mathrm{x}, \mathrm{t})= \\
& =\frac{2^{\mathrm{k}}\left(\mathrm{c}^{2}\right)^{\mathrm{k}}(-1)^{\mathrm{k}}}{\Gamma\left(\left(\frac{\mathrm{n}+1}{2}\right)-\mathrm{k}\right)} \frac{2^{\mathrm{k}}}{\mathrm{c}} \frac{\pi^{\frac{\mathrm{n}}{2}} \pi \Gamma\left(\frac{\mathrm{n}}{2}\right)}{(-1)^{\frac{\mathrm{n}}{2}} \Gamma\left(\frac{\mathrm{n}-1}{2}\right)} \delta(\mathrm{x}, \mathrm{t})= \\
& =\frac{c^{2 k-1}(-1)^{k} 2^{2 k-1}}{\Gamma\left(\left(\frac{n+1}{2}\right)-k\right)} \frac{\pi^{\frac{n}{2}} \Gamma\left(\frac{n}{2}\right)}{(-1)^{\frac{n}{2}} \Gamma\left(\frac{n-1}{2}\right)} \delta(\mathrm{x}, \mathrm{t})
\end{aligned}
$$

Haciendo

$E_{k}(x, t)=\frac{\Gamma\left(\left(\frac{n+1}{2}\right)-k\right)(-1)^{\frac{n}{2}} \Gamma\left(\frac{n-1}{2}\right)}{c^{2 k-1}(-1)^{k} 2^{2 k-1} \pi^{\frac{n}{2}} \Gamma\left(\frac{n}{2}\right)}\left(P_{c}(x, t)\right)_{+}^{\mathrm{k}-\left(\frac{n+1}{2}\right)}$

Si $n$ es par y usando la fórmula (28), se tiene,

$L_{t}^{k}\left\{E_{k}(x, t)\right\}=\delta(\mathrm{x}, \mathrm{t})$.

para $k \geq 1$.

La fórmula (30), significa que $E_{k}(x, t)$ es solución elemental del operador $L_{t}^{k}$ definido en (21).

Nexo Revista Científica Vol. 30, No. 01, pp. 48-59/Junio 2017 
En particular par $k=1$, de (29) se obtiene

$E_{1}(x, t)=-\frac{(-1)^{\frac{n}{2}} \Gamma\left(\frac{n-1}{2}\right)^{2}}{4 c \pi^{\frac{n}{2}} \Gamma\left(\frac{n}{2}\right)}\left(P_{c}(x, t)\right)_{+,}^{-\left(\frac{n-1}{2}\right)}$

Si $n$ es par, es solución elemental del operador

$L_{t}=\frac{\partial^{2}}{\partial t^{2}}-c^{2} \Delta$

Por otra parte de (30) y considerando que $L_{t}^{k}$ y $\delta(\mathrm{x}, \mathrm{t})$ son distribuciones homogéneas entonces la familia de funciones distribucionales $E_{k}(x, t)$ es homogénea. Más aún por cálculo directo $E_{k}(x, t)$ es una distribución homogénea de grado $2 k-(n+1)$ y considerando el teorema de (Donoghue, 1969) se concluye que $E_{k}(x, t)$ es una distribución temperada.

Observamos que la fórmula (29) puede ser escrita de la siguiente forma:

$E_{k}(x, t)=A_{k, n}\left(P_{c}(x, t)\right)_{+}^{\mathrm{k}-\left(\frac{n+1}{2}\right)}$

donde

$A_{k, n}=\frac{\Gamma\left(\left(\frac{n+1}{2}\right)-k\right)(-1)^{\frac{n}{2}} \Gamma\left(\frac{n-1}{2}\right)}{c^{2 k-1}(-1)^{k} 2^{2 k-1} \pi^{\frac{n}{2}} \Gamma\left(\frac{n}{2}\right)}$.

Usando las fórmulas

$\frac{\Gamma(z)}{\Gamma(z-m)}=\frac{-1^{m} \Gamma(-z+m+1)}{\Gamma(1-z)}, y \Gamma\left(\frac{1}{2}+z\right) \Gamma\left(\left(\frac{1}{2}-z\right)=\frac{\pi}{\cos (z \pi)}\right.$

$A_{k, n}$ puede escribirse de la siguiente forma

$A_{k, n}=\frac{\pi^{2}}{2^{2 k-1} k ! \pi^{\frac{n}{2}} c^{2 k-1} \Gamma\left(\mathbf{1}+k-\left(\frac{n+1}{2}\right)\right) \Gamma\left(\mathbf{1}-\left(\frac{n-1}{2}\right)\right) \Gamma\left(\frac{n}{2}\right)}$.

Por otra parte, usando la fórmula $\Gamma(z+m)=z(z+1) \ldots(z+m-1) \Gamma(z), m=1,2, \ldots$ $A_{k, n}$ se puede expresar como

$A_{k, n}=\frac{1}{\left(\Gamma\left(1+\left(\frac{1-n}{2}\right)\right)^{2}\left[\left(\frac{1-n}{2}+1\right) \ldots\left(\frac{1-n}{2}+k-1\right)\right] \Gamma\left(\frac{n}{2}\right)\right.}$

Ahora tomando en cuenta que

$\left[\left(\frac{1-n}{2}+1\right) \ldots\left(\frac{1-n}{2}+k-1\right)\right] \geq\left(\frac{1-n}{2}+1\right)^{k-1}$

para $\mathrm{k}=2,3,4, \ldots$

Nexo Revista Científica Vol. 30, No. 01, pp. 48-59/Junio 2017 
De (35), (36), (37) y (38) se tiene,

$\left|A_{k, n}\right| \leq \frac{\pi}{c} \frac{1}{\mid\left(1+\left.\left(\frac{1-n}{2}\right)\right|^{k-1} \mid\left(\left.\Gamma\left(1+\left(\frac{1-n}{2}\right)\right)\right|^{2} \Gamma\left(\frac{n}{2}\right)\right.\right.} \leq \frac{1}{\mid\left(\left.\Gamma\left(1+\left(\frac{1-n}{2}\right)\right)\right|^{2} \Gamma\left(\frac{n}{2}\right)\right.}$

Por tanto de (33), (39) y considerando que $E_{k}(x, t)$ es una familia de distribuciones temperadas, se concluye que $E_{k}(x, t)$ está acotada para $(x, t) \boldsymbol{\varepsilon} \boldsymbol{R}^{\boldsymbol{n}} \boldsymbol{x} \boldsymbol{R}_{+}$.

Caso 2: $n$ impar. De (25) y haciendo $m=n+1$ para $n$ impar se tiene,

$$
\begin{aligned}
& L_{t}^{k}\left(P_{c}(x, t)\right)_{+,}^{\lambda+k}=\left(2 c^{2}\right)^{k}(\boldsymbol{\lambda}+\mathbf{1}) . .(\boldsymbol{\lambda}+\boldsymbol{k})(\mathbf{2} \boldsymbol{\lambda}+\boldsymbol{n}+\mathbf{1}) \ldots(\mathbf{2} \boldsymbol{\lambda}+\boldsymbol{n}+\mathbf{2} \boldsymbol{k}+\mathbf{1})\left(P_{c}(x, t)\right)_{+,}^{\lambda}= \\
& =\frac{\left(2 c^{2}\right)^{k}(\boldsymbol{\lambda}+\mathbf{1}) . .(\boldsymbol{\lambda}+\mathbf{k})(2 \boldsymbol{\lambda}+\mathbf{n}+\mathbf{1}) \ldots(2 \boldsymbol{\lambda}+\mathbf{n}+\mathbf{2} \mathbf{k}-2)(2 \boldsymbol{\lambda}+\mathbf{m})^{2}\left(P_{c}(x, t)\right)_{+}^{\lambda}}{2\left(\lambda+\frac{m}{2}\right)} \\
& =\left(2 c^{2}\right)^{k}(\boldsymbol{\lambda}+\mathbf{1}) . .(\boldsymbol{\lambda}+\mathbf{k})(2 \boldsymbol{\lambda}+\mathbf{n}+\mathbf{1}) \ldots(\mathbf{2} \boldsymbol{\lambda}+\mathbf{n}+\mathbf{2} \mathbf{k} \\
& -2)(2 \lambda+\mathbf{m})^{2} \cdot \frac{1}{2\left(\lambda+\frac{m}{2}\right)}\left(P_{c}(x, t)\right)_{+}^{\lambda}
\end{aligned}
$$

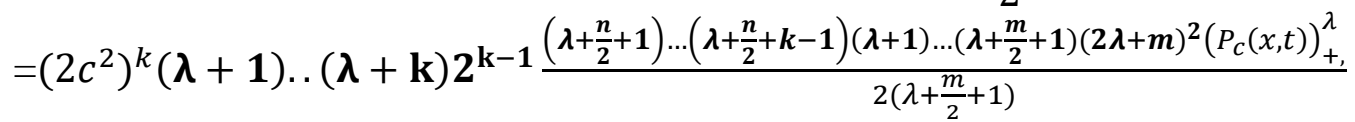

$$
\begin{aligned}
& =\left(2 c^{2}\right)^{k} \frac{\Gamma(\lambda+\mathbf{1}+\boldsymbol{k})}{2 \Gamma(\boldsymbol{\lambda}+\mathbf{1})} \cdot \frac{\left.2^{k-1}\left(\lambda+\frac{\boldsymbol{n}}{\mathbf{2}}+\mathbf{1}\right) \ldots \lambda+\frac{\boldsymbol{n}}{\mathbf{2}}+\boldsymbol{k}-\mathbf{1}\right)}{\Gamma\left(\lambda+\frac{\boldsymbol{m}}{\mathbf{2}}+\mathbf{1}\right)} .(\lambda+\mathbf{1}) \ldots\left(\lambda+\frac{\boldsymbol{m}}{\mathbf{2}}+\mathbf{1}\right) \Gamma(\lambda \\
& \text { +1) }(2 \lambda+\boldsymbol{m})^{2}\left(P_{c}(x, t)\right)_{+}^{\lambda} \\
& =\left[\left(2 c^{2}\right)^{k} \frac{\Gamma(\lambda+\mathbf{1}+k)}{2 \Gamma(\lambda+\mathbf{1})} \cdot\left(\lambda+\frac{\boldsymbol{n}}{2}+\mathbf{1}\right) \ldots\left(\lambda+\frac{\boldsymbol{n}}{2}+\boldsymbol{k}-\mathbf{1}\right)(\lambda+\mathbf{1}) \ldots\left(\lambda+\frac{\boldsymbol{m}}{2}+\mathbf{1}\right) 2^{k-1} 2^{2}\left(\lambda+\frac{m}{2}\right)\right] \\
& .\left(P_{c}(x, t)\right)_{+,}^{\lambda}\left(\lambda+\frac{m}{2}\right)
\end{aligned}
$$

Por otra parte, el desarrollo de $\left(P_{c}(x, t)\right)_{+,}^{\lambda+k}$ en series de Taylor alrededor de $\lambda=-\frac{(n+1)}{2}=-\frac{m}{2}$ está dado por:

$$
\begin{aligned}
& \left(P_{c}(x, t)\right)_{+,}^{\lambda+k}=\left(P_{c}(x, t)\right)_{+,}^{k-\frac{m}{2}}+\left(P_{c}(x, t)\right)_{+,}^{k-\frac{m}{2}} \ln \left(\left(P_{c}(x, t)\right)_{+,}^{k-\frac{m}{2}}\right)\left(\lambda+\frac{m}{2}\right)= \\
& =\left(\sum_{j \geq 0}\left(\left(P_{c}(x, t)\right)_{+,}^{k-\frac{m}{2}} \ln ^{j}\left(\left(P_{c}(x, t)\right)_{+,}^{k-\frac{m}{2}}\right)\right)\left(\lambda+\frac{m}{2}\right)\right)
\end{aligned}
$$

para $k>\frac{m}{2}$

Comparando los coeficientes de $\left(\lambda+\frac{m}{2}\right)$ en ambas ecuaciones (40) y (41)

$$
\begin{gathered}
L_{t}^{k}\left(P_{c}(x, t)\right)_{+,}^{k-\frac{(n+1)}{2}}=\lim _{\lambda \rightarrow-\frac{(n+1)}{2}} L_{t}^{k}\left(P_{c}(x, t)\right)_{+,}^{\lambda+k}= \\
=\lim _{\lambda \rightarrow-\frac{(n+1)}{2}}\left(2 c^{2}\right)^{k} \frac{1}{2} \cdot \Gamma(\boldsymbol{\lambda}+\mathbf{1}+\boldsymbol{k}) \cdot(\boldsymbol{\lambda}+\mathbf{1}) \ldots\left(\boldsymbol{\lambda}+\frac{\boldsymbol{m}}{\mathbf{2}}-\mathbf{1}\right) .
\end{gathered}
$$

Nexo Revista Científica Vol. 30, No. 01, pp. 48-59/Junio 2017 


$$
\left.\lim _{\lambda \rightarrow-\frac{(n+1)}{2}}\left(\boldsymbol{\lambda}+\frac{\boldsymbol{n}}{\mathbf{2}}+\mathbf{1}\right) \ldots\left(\boldsymbol{\lambda}+\frac{\boldsymbol{n}}{\mathbf{2}}+\boldsymbol{k}\right)-\mathbf{1}\right) 2^{k-1} 2^{2} .
$$

$. \lim _{\lambda \rightarrow-\frac{(n+1)}{2}}\left[\left(\lambda+\frac{m}{2}\right) \cdot\right]\left(P_{c}(x, t)\right)_{+}^{\lambda}=2^{2 k} c^{2 k}\left(k-\frac{(n+1)}{2}\right) !(k-1) !(-1)^{\frac{(n+1)}{2}-1}\left(\frac{(n+1)}{2}-\right.$ 1)! $\frac{\Gamma\left(k-\frac{1}{2}\right)}{\Gamma\left(-\frac{1}{2}\right)}$

$\operatorname{Res}_{\lambda=-\frac{(n+1)}{2}}\left(P_{c}(x, t)\right)_{+}^{\lambda}$

Por otra parte, de (Gelfand y Shilov, 1964) y usando la fórmula

$\delta_{1}^{(s)}(H)=\frac{2(-1)^{s-1}(-1)^{\frac{q+1}{2}} \pi^{\frac{n}{2}-1}}{4^{s-\frac{n}{2}+1}\left(s-\frac{n}{2}+1\right) !}\left[\psi\left(\frac{p}{2}\right)-\psi\left(\frac{n}{2}\right)\right] L^{s-\frac{n}{2}+1} \boldsymbol{\delta}$ (Aguirre, 2000)

si $p+q=n$ es la dimensión del espacio, $p$ y $q$ ambos impares.

donde

$H=H\left(x_{1, \ldots} x_{n}\right)=x_{1}^{2}+\cdots+x_{p}^{2}-x_{p+1}^{2}-\cdots-x_{p+q}^{2}$

$L=\frac{\partial^{2}}{\partial x_{1}^{2}}+\cdots+\frac{\partial^{2}}{\partial x_{p}^{2}}-\frac{\partial^{2}}{\partial x_{p+1}^{2}}-\cdots \frac{\partial^{2}}{\partial x_{p+q}^{2}}$

$\psi(x)=\frac{\Gamma(x)}{\Gamma(x)}$

$\psi(l)=-c+1+\frac{1}{2}+\cdots+\frac{1}{l-1}, l=2,3, \ldots$

$\psi\left(l+\frac{1}{2}\right)=-c-2 \ln 2+2\left(1+\frac{1}{3}+\cdots+\frac{1}{2 l-1}\right), l=1,2,3, \ldots$

y $c$ constante de Euler, para el caso $p=1$ y $q=1$ considerando la fórmula (43), se tiene la siguiente la siguiente fórmula:

$\operatorname{Res}_{\lambda=-\frac{d}{2}-s, s=0,1,2, \ldots}\left(P_{c}(x, t)\right)_{+,}^{\lambda}=\frac{(-1)(-1)^{\frac{d}{2}} \pi^{\frac{d}{2}-1}}{4^{s} s ! !}\left[\psi\left(\frac{1}{2}\right)-\psi\left(\frac{d}{2}\right)\right] L^{s} \boldsymbol{\delta}$

donde $d$ es la dimensión par del espacio.

Haciendo $s=0$ en (49), se tiene,

$\cdot \operatorname{Res}_{\lambda=-\frac{d}{2}}\left(P_{c}(x, t)\right)_{+,}^{\lambda}=(-1)(-1)^{\frac{d}{2}} \pi^{\frac{d}{2}-1}\left[\psi\left(\frac{1}{2}\right)-\psi\left(\frac{d}{2}\right)\right] \boldsymbol{\delta}(\mathrm{x}, \mathrm{t})$

Si $d$ es par.

De (42) y (50) se obtiene la siguiente fórmula:

Nexo Revista Científica Vol. 30, No. 01, pp. 48-59/Junio 2017 


$$
\begin{aligned}
& L_{t}^{k}\left(P_{c}(x, t)\right)_{+}^{k-\frac{(n+1)}{2}}=2^{2 k} c^{2 k}\left(k-\frac{(n+1)}{2}\right) !(k-1) !(-1)^{\frac{(n+1)}{2}-1}\left(\frac{(n+1)}{2}-1\right) ! \frac{\Gamma\left(k-\frac{1}{2}\right)}{\Gamma\left(-\frac{1}{2}\right)} . \\
& (-1)(-1)^{\frac{n+1}{2}} \pi^{\frac{n+1}{2}-1}\left[\psi\left(\frac{1}{2}\right)-\psi\left(\frac{n+1}{2}\right)\right] \boldsymbol{\delta}(\mathrm{x}, \mathrm{t})
\end{aligned}
$$

si $n$ es impar y $k-\frac{(n+1)}{2} \geq 0$.

Por tanto haciendo

$$
\overline{E_{k}(x, t)}=\frac{\left(P_{c}(x, t)\right)_{+,}^{k-\frac{(n+1)}{2}}}{2^{2 k} c^{2 k}\left(k-\frac{(n+1)}{2}\right) !(k-1) !(-1)^{\frac{(n+1)}{2}}-1\left(\frac{(n+1)}{2}-1\right) ! \frac{\Gamma\left(k-\frac{1}{2}\right)}{\Gamma\left(-\frac{1}{2}\right)}(-1)(-1)^{\frac{n+1}{2}} \pi^{\frac{n+1}{2}}-1\left[\psi\left(\frac{1}{2}\right)-\psi\left(\frac{n+1}{2}\right)\right]}
$$

de (51) se obtiene que $\overline{E_{k}(x, t)}$ es solución elemental de $L_{t}$ iterado $k$ veces si $n$ es impar y $k \geq$ $\frac{(n+1)}{2}$.

Ahora considerando las fórmulas (47) y (48) se tiene

$$
\psi\left(\frac{1}{2}\right)-\psi\left(\frac{n+1}{2}\right)=\left\{\begin{array}{c}
-2 \ln 2 \text { si } n=1 \\
-\left(2 \ln 2+1+\frac{1}{2}+\cdots+\frac{1}{\frac{n-1}{2}} \text { si } n=3,5,7, \ldots\right.
\end{array}\right.
$$

Usando (53), $\overline{E_{k}(x, t)}$ puede ser escrita de la siguiente forma:

$$
\overline{E_{k}(x, t)}=\left\{\begin{array}{c}
\frac{\left(P_{c}(x, t)\right)_{+,}^{k-1}}{2^{2 k} c^{2 k}(k-1) !(k-1) ! 2 \ln 2 \frac{\Gamma\left(k-\frac{1}{2}\right)}{\Gamma\left(-\frac{1}{2}\right)}} \sin =1 y k \geq 1 \\
y \\
\frac{\left(P_{c}(x, t)\right)_{+,}^{k-\frac{(n+1)}{2}}}{2^{2 k} c^{2 k}\left(k-\frac{(n+1)}{2}\right) !(k-1) !(-1)^{\frac{(n+1)}{2}}-1\left(\frac{(n+1)}{2}-1\right) ! \frac{\Gamma\left(k-\frac{1}{2}\right)}{\Gamma\left(-\frac{1}{2}\right)}(-1)(-1)^{\frac{n+1}{2}} \pi^{\frac{n+1}{2}-1}\left[\psi\left(\frac{1}{2}\right)-\psi\left(\frac{n+1}{2}\right)\right]} \sin =3,5,7, \ldots y k \geq \frac{(n+1)}{2} .
\end{array}\right.
$$

\section{APLICACIONES}

Sea $E_{k}(x, t)$ la familia de soluciones e elementales del operador diferencial iterado $k$ veces $L_{t}^{k}$ definido por (32), esto es

$L_{t}^{k}\left\{E_{k}(x, t)\right\}=\delta(\mathrm{x}, \mathrm{t})$, si $n$ es par

donde

$$
L_{t}^{k}=\left\{\frac{\partial^{2}}{\partial t^{2}}-c^{2} \Delta\right\}^{k}, k=1,2, \ldots
$$


Consideremos la ecuación diferencial no lineal.

$L_{t}^{k} u_{k}(x, t)=f\left(x, t, u_{k}(x, t)\right)$. Para $k=1,2, \ldots$

donde $L_{t}^{k}$ está definido por (56) y $f\left(x, t, u_{k}(x, t)\right) \varepsilon^{\prime} D^{\prime}\left(\Gamma^{+}\right)$(espacio de distribuciones definido en $\Gamma^{+}$) (Schwartz, 1957 ; Choquet, 1973) entonces usando (55) se tiene,

$L_{t}^{k}\left\{u_{k}(x, t) * f\left(x, t, u_{k}(x, t)\right)\right\}=L_{t}^{k} u_{k}(x, t) * f\left(x, t, u_{k}(x, t)\right)=\delta(\mathrm{x}, \mathrm{t}) * f=f$

$\mathrm{y}$

$E_{k}(x, t) * L_{t}^{k} u_{k}(x, t)=L_{t}^{k} E_{k}(x, t) * u_{k}(x, t)=\delta(\mathrm{x}, \mathrm{t}) * u_{k}(x, t)=u_{k}(x, t)$

Por tanto de (58) y (59) la solución de la ecuación diferencial no lineal (57) está dada por:

$u_{k}(x, t)=E_{k}(x, t) * L_{t}^{k} u_{k}(x, t)=E_{k}(x, t) * f\left(x, t, u_{k}(x, t)\right)$

si $n$ es par.

Observamos que el soporte de $\left\{E_{k}(x, t)\right\} \subset \Gamma_{+}$, entonces $\left\{E_{k}(x, t)\right\} \in{ }^{\prime} D^{\prime}\left(\Gamma^{+}\right)$y tomando en cuenta (Schwartz, 1957), se concluye que $u_{k}(x, t) \in{ }^{\prime} D^{\prime}\left(\Gamma^{+}\right)$para $k=1,2,3, \ldots$

En forma similar usando (51) y (52) se obtiene que la solución de la ecuación no lineal (57) está dada por:

$V_{k}(x, t)=\overline{E_{k}(x, t)}(x, t) * f\left(x, t, V_{k}(x, t)\right)$

si $n$ es par y $k \geq \frac{n+1}{2} \ldots$

En particular haciendo $k=1$ en (60) y considerando (33) y (34) se obtiene

$$
\begin{aligned}
& G(x, t)=u_{1}(x, t)=E_{1}(x, t) * f\left(x, t, u_{1}(x, t)\right)= \\
& =\int_{t} t>0 \int_{R^{n}} E_{1}(x-y, t) f(x, t, u(x-y, t) d y d t= \\
& =\int_{t>0} \int_{R^{n}} E_{1}(x, t) f(x-y, t, u(x-y, t) d y d t
\end{aligned}
$$

como solución de la ecuación diferencial no lineal dada en(57) para el caso $k=1$, donde

$$
\begin{aligned}
& E_{1}(x, t)=A_{1, n}\left(P_{c}(x, t)\right)_{+,}^{1-\left(\frac{n+1}{2}\right)}=\frac{\Gamma\left(\left(\frac{n+1}{2}\right)-1\right)(-1)^{\frac{n}{2}} \Gamma\left(\frac{n-1}{2}\right)}{(-1) 2 c \pi^{\frac{n}{2}}}\left(P_{c}(x, t)\right)_{+,}^{-\left(\frac{n-1}{2}\right)}= \\
& ==\frac{\left.\left(\Gamma\left(\left(\frac{n+1}{2}\right)-1\right)\right)^{2}(-1)^{\frac{n}{2}}\right)}{(-1) 2 c \pi^{\frac{n}{2}}}\left(P_{c}(x, t)\right)_{+,}^{-\left(\frac{n-1}{2}\right)}= \\
& =\frac{\Pi^{2}}{2 c \pi^{\frac{n}{2}}(-1)^{\frac{n}{2}} \Gamma\left(2-\left(\frac{n+1}{2}\right)\right) \Gamma\left(1-\left(\frac{n-1}{2}\right.\right.}\left(P_{c}(x, t)\right)_{+}^{-\left(\frac{n-1}{2}\right)}
\end{aligned}
$$


si $n$ es par.

Observamos que haciendo $n=3$ en (61) y considerando (53) y (54), se tiene

$$
\begin{aligned}
& V_{k}(x, t)=\overline{E_{k}(x, t)}(x, t) * f\left(x, t, V_{k}(x, t)\right)= \\
& \frac{\left(P_{c}(x, t)\right)_{+,}^{k-2} * f\left(x, t, V_{k}(x, t)\right)}{2^{2 k} c^{2 k}(k-2) !(k-1) ! \Pi(-1)(2 L N 2+1)}= \\
& \int_{t>0} \int_{R^{n}}\left(P_{c}(x-y, t)\right)_{+,}^{k-2} f\left(x, t, V_{k}(x, t) d y d t=\right. \\
& =\frac{1}{2^{2 k} c^{2 k}(k-2) !(k-1) ! \Pi(-1)(2 L N 2+1)} . \\
& \int_{t>0} \int_{R^{n}}\left(P_{c}(x, t)\right)_{+,}^{k-2} f\left(x-y, t, V_{k}(x-y, t) d y d t\right.
\end{aligned}
$$

si $k \geq 2$.

Podemos observar que (Wanchak, 2016), usando la transformada de Fourier obtiene que

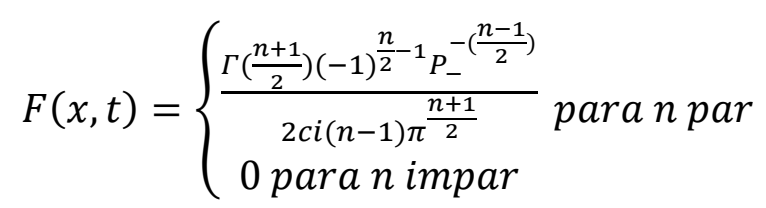

Es solución elemental del operador $L_{t}$ definido por (32), donde

$$
P_{-}^{\lambda}=\left\{\begin{array}{c}
\left(-P^{\lambda}\right) \text { si } P<0 \\
y \\
0 \text { si } P \leq 0
\end{array}\right.
$$

y $P=H$ (ver fórmula (44)).

(Wanchak, 2016) introduce la ecuación no lineal para el caso $k=1$ y bajo condiciones especiales para $f(x, t, F(x, t))$ y obtiene que $u_{1}(x, t)=F(x, t) * \mathrm{f}(\mathrm{x}, \mathrm{t}, \mathrm{F}(\mathrm{x}, \mathrm{t}))$ es solución única de la ecuación(57) para el caso k=1.

Finalmente de (25) y (27) se tiene que $L_{t}^{k}\left\{\left(P_{c(x, t)}{ }^{k-\frac{(n+1)}{2}}\right)=0\right.$ si $k \leq \frac{n+1}{2}-1=\frac{n-1}{2}$ si $n$ es impar. Por tanto $P_{c(x, t)}{ }^{k-\frac{(n+1)}{2}}$ es solución de la ecuación homogénea (57).

\section{CONCLUSIONES}

En este artículo se generalizan fórmulas vinculadas con el operador de las ondas. Es conocido en la teoría de ecuaciones en derivadas parciales la ecuación de la onda en una y varias dimensiones. Los resultados de este artículo generalizan ese tipo de soluciones y planteamos un método general para generar soluciones de ese tipo de ecuaciones en derivadas parciales. El método permite que a través de encontrar soluciones elementales podamos encontrar la solución general de ecuaciones incluso no lineales. Las 
soluciones quedan expresadas a través de una integral por medio de la operación de convolución. También se obtiene para el caso de dimensión impar una familia de soluciones homogéneas para la ecuación de la onda generalizada. Los fundamentos matemáticos de los resultados de este artículo están soportado en la teoría de funciones generalizadas ó teoría de distribuciones.

\section{SEMBLANZA DE LOS AUTORES}

Manuel Antonio Aguirre: Obtuvo el grado de licenciado en Ciencias de la Educación Orientación Matemática en la UNAN-Managua, Nicaragua. Desarrolló sus estudios de Licenciatura y Doctorado en Ciencias Matemáticas en la Facultad de Ciencias Exactas y Naturales de la Universidad de Buenos Aires, Argentina. Es miembro correspondiente de la Academia de Ciencias de Buenos Aires, Argentina. La UNAN-Managua en el año 2010, le otorgó en título de "Doctor Honoris Causa". Actualmente es Profesor Titular del departamento de Matemática de la Facultad de Ciencias Exactas de la Universidad Nacional del Centro y Director del Núcleo Consolidado de Matemática Pura y Aplicada(NuCOMPA).

Emilio Andrés Aguirre: Obtuvo el grado de licenciado en Ciencias Matemáticas en la Facultad de Ciencias Exactas de la Universidad Nacional del Centro, Argentina. Desarrollo sus estudios de Maestría y Doctorado en la Universidad de Valladolid España. Actualmente es docente del departamento de Matemática de la Facultad de Ciencias Exactas de la Universidad Nacional del Centro e integra el proyecto " Productos de Distribuciones y transformaciones generalizada" del NuCOMPA.

\section{REFERENCIAS}

Aguirre, M.A. (2000). Proportionality ofk-derivative of Dirac delta in the hypercone, Mathematica Balkanica, volume 24, Book 3-4, pp.253-263.

Choquet, B.Y. 1973). Distribution theorie et problems, Masson, Paris 1973.

Donoghue, W.A., Distributions and Fourier transform, Academic Press, 1969.

Erdelyi, A., (1964), Higher transcendental Functions, Vol.1, McGraw-Hill, New York, U.S.A. Gelfand, I. and Shilov, G. (1964), generalized Functions, Vol. 1, Academic Press, New York, U.S.A.

Schwartz,L.,(1957), Théorie des distributions, Herman, Paris, Francia.

Wanchat, S. (2016), On nolinear wave equation, (to appear). 